“C 2017 IEEE. Personal use of this material is permitted. Permission from IEEE must be obtained for all other uses, in any current or future media, including reprinting/republishing this material for advertising or promotional purposes, creating new collective works, for resale or redistribution to servers or lists, or reuse of any copyrighted component of this work in other works." 


\title{
Core Loss Measurement under Elliptical Loci of Magnetic Flux Density
}

\author{
Ashraf Rohanim Asari ${ }^{1,2^{*}}$, Youguang Guo ${ }^{1}$ and Jianguo Zhu ${ }^{1}$ \\ ${ }^{1}$ Faculty of Engineering and Information Technology, University of Technology Sydney (UTS), Australia \\ ${ }^{2}$ Instrumentation and Control Engineering Section, University Kuala Lumpur (MITEC), Malaysia \\ Emails: *ashraf@unikl.edu.my, youguang.guo-1@uts.edu.au, jianguo.zhu@uts.edu.au
}

\begin{abstract}
Nowadays, rotating machines are widely used in most industries. Because of that, magnetic properties of soft magnetic material are actively studied under 1-D and 2-D magnetic flux excitations in order to estimate the total core loss produced by the material during the magnetization. SOMALOY 700 material, a soft magnetic composite material, is considered in this paper. 2-D magnetic measurement has been conducted by controlling magnetic flux densities to be in ellipse shape by using LabVIEW software. The core loss has been computed by using MathCAD software. The performance of this material at some frequencies is compared by plotting the loss curves in the same graph.
\end{abstract}

Index Terms-Core loss measurement, elliptical loci, magnetic flux density.

\section{INTRODUCTION}

In rotating electric machines, the analytical techniques are varied and quite different, depending on the type and the objective of the machine. Generally, in an electrical machine, there are three important components in operating the machines such as the stator core, winding coils, and rotor core.

The stator and rotor cores are made from magnetic material to aid the production of magnetic flux density. The rotating magnetic fields are generated when three-phase AC currents flow through the winding coils. The fields pass through the air gap and the cores.

To predict the actual core loss that has been produced by the actual electrical motor, the magnetic properties of material should be investigated under similar mode of magnetic flux density propagation which is rotating. Due to that, the magnetic flux densities are produced to be elliptical by controlling the magnitude, direction, and phase angle of individual magnetic field components.

It is very important to properly identify and model the magnetic properties of material under variation of magnetic flux densities in order to be employed in designing and simulation of electric devices. With this valuable information, the performance of electric devices can be well predicted.

\section{Magnetic Property Testing System}

The UTS 3-D magnetic property testing system has successfully been developed by Zhu, et al. in 2001. This 3-D tester can magnetize the cubic samples of soft magnetic materials [1]-[4]. To be operated, the tester is attached to a feedback control system which contains a control unit and three-channel power amplifier for generating three different axes of magnetic fluxes [2]-[6].

The voltage waveforms of magnetic flux density for the $\mathrm{x}-, \mathrm{y}-$ and $\mathrm{z}$-components are generated and exported to a three-channel power amplifier, which feeds the excitation winding coils of the tester, through three isolated channels of the $\mathrm{A} / \mathrm{D}$ and $\mathrm{D} / \mathrm{A}$ board. The $\mathrm{x}-, \mathrm{y}-$ and $\mathrm{z}$-components of $\mathrm{B}$ (magnetic flux density) and $\mathrm{H}$ (magnetic field strength) sensing coils generate voltage signals, which are collected by six independent input channels of the $\mathrm{A} / \mathrm{D}$ and $\mathrm{D} / \mathrm{A}$ board. The signals are used to determine the relationship of $\mathrm{B}$ and $\mathrm{H}$ in order to calculate the core loss of the sample. The signals of B are also used for feedback control of the waveforms [2][7] by using the LabVIEW software.

\section{CALibration OF SEnsing CoILs}

To ensure the accuracy in measuring the magnetic properties of SOMALOY 700 material, the sensing coils that are used in detecting the magnetic flux density during the measurement, are gone through calibration process in the middle of a long solenoid. The calibration also offers the consistency with other and repetitive measurements.

The electromotive forces of the sensing coils are induced inside the solenoid when there is a changing of magnetic flux as stated by Faraday's Law. In this experiment, maximum magnetic field created by a solenoid will be measured. The magnetic flux density for a long solenoid of many turns is given as,

$$
\mathrm{B}=\mu_{0} \frac{N_{s}}{l} \mathrm{I}
$$

where $B$ is the flux density which is nearly uniform, $\mathrm{N}_{\mathrm{s}}$ is the number of turns, $l$ is the total length of the solenoid, and $I$ is the current passing through the solenoid coils. The magnetic flux over some area $\mathrm{A}$ is,

$$
\emptyset=\mathrm{B} \cdot \mathrm{A}
$$

Faraday's Law relates the time rate of change of the flux, $\mathrm{d} \emptyset / \mathrm{dt}$, to the electromotive force (emf) voltage, $\varepsilon$ as below,

$$
\varepsilon=\mathrm{N}_{\mathrm{s}} \mathrm{A}_{\mathrm{s}} \frac{\mathrm{dB}}{\mathrm{dt}}
$$

In (3), the sensing coil is stationary and perpendicular to the penetration of $\mathrm{B}$. If the $\mathrm{AC}$ current in the solenoid has a frequency $f, I=I_{0} \sin (\omega t)$, then the magnetic flux density at the center of the solenoid is $B=B_{0} \sin (\omega t)$. Thus, the sensing coil induces an emf voltage whose amplitude $\varepsilon_{0}$ is proportional to the frequency $\omega$ and the amplitude $B_{o}$ of the magnetic flux density.

$$
\varepsilon_{0}=\omega N_{p} A_{p} B_{0}
$$


If the sensing coil is located inside a solenoid and the induced emf of the coil is given by:

$$
\varepsilon_{0}=2 \pi f N_{p} A_{p} \mu_{0} n_{s} I_{0}
$$

where $2 \pi \mathrm{f}=\omega, n_{s}$ is the number of turns per unit length of the solenoid. It can be seen that the emf of the sensing coil is proportional to both the frequency $f$ and the amplitude $I_{o}$ of the AC current in the solenoid.

A circuit diagram of the experiment is shown in Fig. 1. The AC voltage supply produces a sine-wave voltage which drives an AC current through the solenoid. The solenoid, with $0.8 \mathrm{~m}$ length and $0.11 \mathrm{~m}$ diameter, has 939 turns and $13.3 \mathrm{mH}$ of inductance. The change of magnetic fluxes induces the $\mathrm{B}$ and $\mathrm{H}$ sensing voltages in the long solenoid. The operational amplifiers are needed to amplify the sensing voltages before being measured by the LabVIEW software.

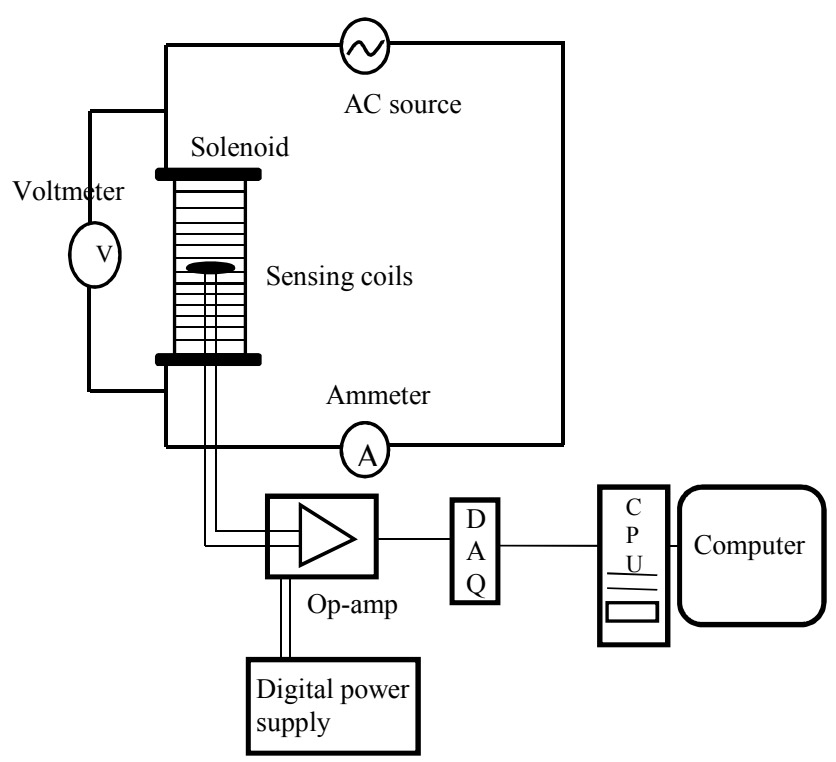

Fig. 1. Circuit diagram of calibration process.

The magnetic flux density $B$, that is generated by solenoid, and induced emf of the sensing coil, $\varepsilon_{0}$ will be used in obtaining the sensing box coefficients, $K_{H}$ and $K_{B}$.

The plotted sensing voltages $V$, along $\mathrm{x}, \mathrm{y}$ and $\mathrm{z}$ axes versus alternating currents $I$ that pass through the solenoid, are described in Fig. 2. It can be seen that the emf of the $B$ and $H$ sensing coils is directly proportional to amplitude of the $\mathrm{AC}$ current in the solenoid as stated in (5).

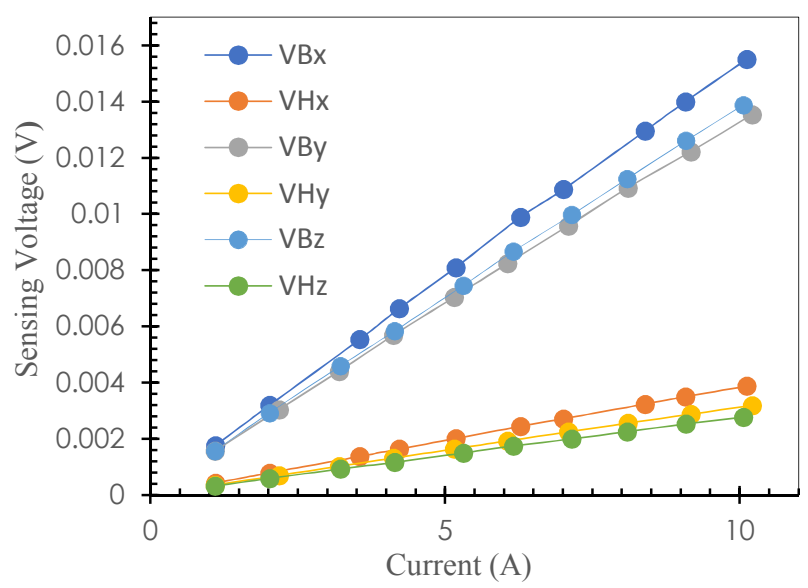

Fig. 2. Induced voltages of $\mathrm{B}$ and $\mathrm{H}$ coils during the calibration process.

The results which are obtained by averaging over several measurements are $K_{H x}=0.0012, K_{H y}=0.0010, K_{H z}=0.0009$, $K_{B x}=0.0048, K_{B y}=0.0042$, and $K_{B z}=0.0043$.

\section{EXPERIMENTAL SETUP}

The $20 \times 20 \times 20 \mathrm{~mm}$ of $7.5 \mathrm{~kg} / \mathrm{m}^{3}$ SOMALOY 700 cubic samples from Hoganas are with six guarding pieces which are cut to be $2 \times 20 \times 20 \mathrm{~mm}$.

To obtain the uniformity and high accuracy of magnetic flux density $B$, the $0.05 \mathrm{~mm}$ diameter of enameled copper wire is used for both sensing coils. The 200 turns of $H$ sensing coil are wound around $0.6 \mathrm{~mm}$ thickness epoxy resin board. The round $B$ sensing coil with $6 \mathrm{~mm}$ diameter has been embedded in the center of the $H$ coil, which has been wound for 60 turns.

Fig. 3 shows the $B$ and $H$ sensing coils which are located on the surfaces of the cubic sample. The tiny sensing coils are protected by applying epoxy resin. Epoxy glue coats the coils to prevent the coils.

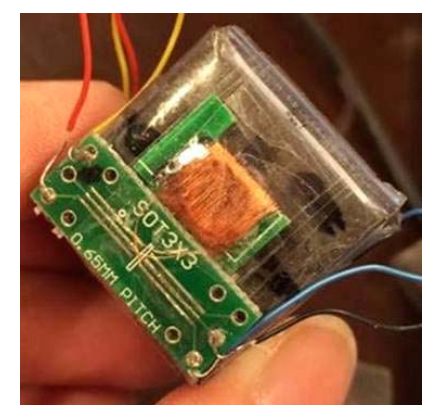

Fig. 3. SOMALOY 700 cubic sample with sensing coils.

The sensing box was built to hold the guarding pieces, sensing coils, and sample together. Fig. 4 shows the sample of SOMALOY 700 with its guarding pieces which have been held by the sensing box. 


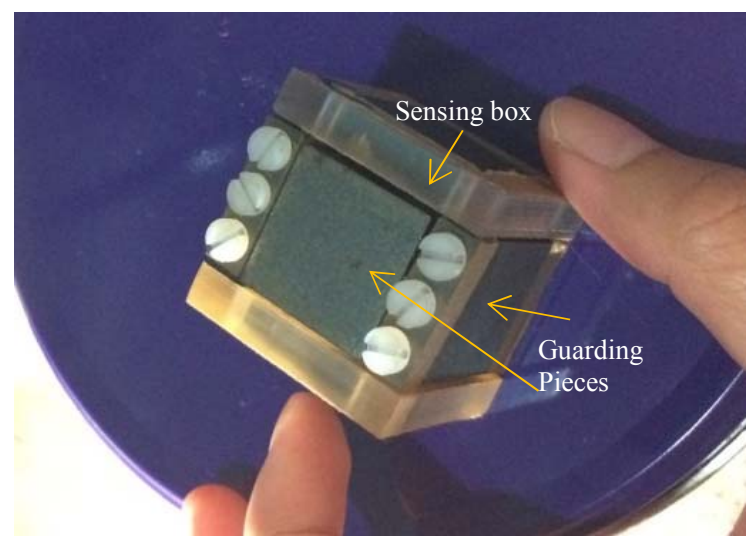

Fig. 4. An acrylic sensing box with guarding pieces, holding the sensing coils to be located on the surfaces of the cubic sample.

\section{Magnetic Properties MEASUREMENT}

The elliptical $B$ loci are examined by producing major and minor axis during the measurement. To produce elliptical $B$ loci that lie in the xoy plane, the magnitudes of $B_{x}$ and $B_{y}$ should be controlled properly.

DAQ devices from National Instrumentation (NI) have been used as an interface to generate and control the magnetic flux density waveforms by using LabVIEW software.

In detail, the magnitudes and phase angle between $B_{x}$ and $B_{y}$ are controlled in front panel of LabVIEW, which is able to produce AC voltage waveforms in the $x$ and $y$-directions. Then, the waveforms are amplified by using power amplifier (model: AM3002), which can amplify 63.5 times or $36 \mathrm{~dB}$ before generating the magnetic flux density with the excitation winding coils in the 3-D tester [8].

Thus, the rotating core loss with elliptical $B$ loci can be predicted by considering the equation below

$$
P_{t}=R_{B} P_{r}+\left(1-R_{B}\right)^{2} P_{a}
$$

where $P_{\alpha}$ is the core loss with an alternating $B, P_{r}$ is the core loss with a circular $B$ and $R_{B}$ is the axis ratio that can be determined by dividing $B_{m i n}$ by $B_{m a j}$. In this case, $B_{m a j}=B_{p}$ is the peak value of alternating $B$.

Elliptical magnetic flux densities are controlled to lie in the xoy plane at $50 \mathrm{~Hz}, 100 \mathrm{~Hz}$ and $1000 \mathrm{~Hz}$ of operating frequency, respectively. Each measurement under certain frequency is performed twice for representing the $\mathrm{x}$ and $\mathrm{y}$ major. Both measurements are compared by plotting the core loss curve at each frequency.

\section{EXPERIMENTAL RESULTS}

The loci of magnetic flux density vector are controlled to be elliptical in order to resemble the actual magnetic flux excitation in rotating electrical machines. The measurement has been conducted under elliptical magnetic field on the xoy plane. The $B$ loci at $50 \mathrm{~Hz}, 100 \mathrm{~Hz}$ and $1000 \mathrm{~Hz}$ that lie in the xoy plane are illustrated in Fig. 5. Fig. 5 (a) shows the $B$ loci with $B_{x}$ as the major axis and $B_{y}$ as the minor axis. Fig. 5 (b) shows the $B$ loci at three different frequencies with $B_{y}$ as the major axis and $B_{x}$ as the minor axis.

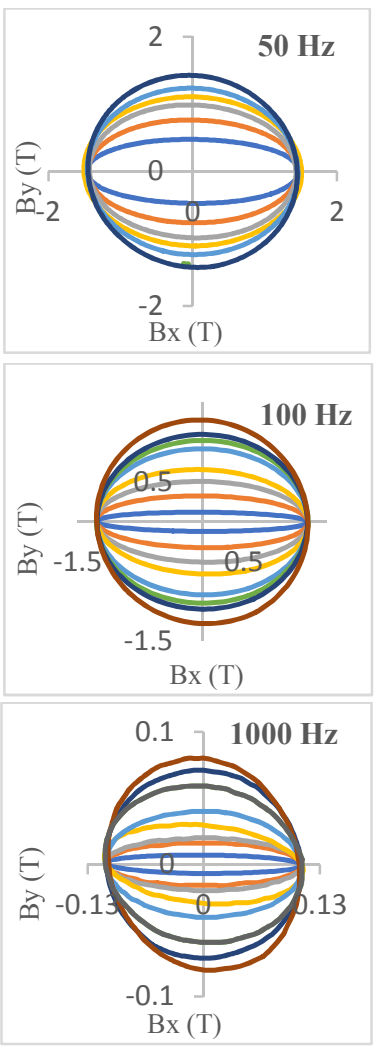

(a)
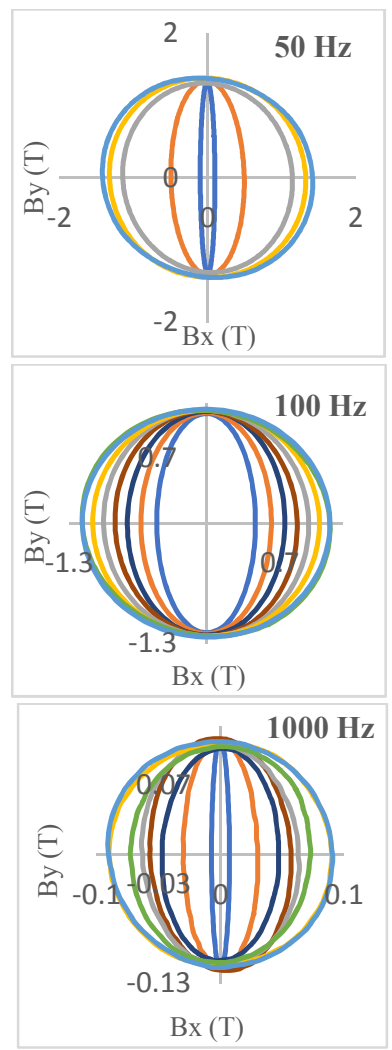

(b)
Fig. 5. B loci at $50 \mathrm{~Hz}, 100 \mathrm{~Hz}$ and $1000 \mathrm{~Hz}$ of operating frequency: a) $x$ major, b) $y$-major

Figs. 5 (a) and (b) show that both B loci for major axes $B_{x}$ and $\mathrm{B}_{\mathrm{y}}$ lie in the same magnetization plane. As can be seen, the $\mathrm{B}$ loci are distorted at high frequency $(1000 \mathrm{~Hz})$. It may be caused by the movement of rotating domain that is increased during the magnetization which will portray the anisotropy property when there is involvement of high magnetic field in different directions [9], [10].

At $1000 \mathrm{~Hz}$ of magnetization, the shape of elliptical B loci becomes shaky due to the coupling between core poles. This also leads to the noise production but it does not contribute to the core losses [11]. 
The corresponding $\mathrm{H}$ loci are plotted in Fig. 6, where Fig. 6 (a) illustrates the $\mathrm{H}$ loci when $\mathrm{B}_{\mathrm{x}}$ acts as the major axis and Fig.6 (b) describes the $\mathrm{H}$ loci when $\mathrm{B}_{\mathrm{y}}$ is the major axis.

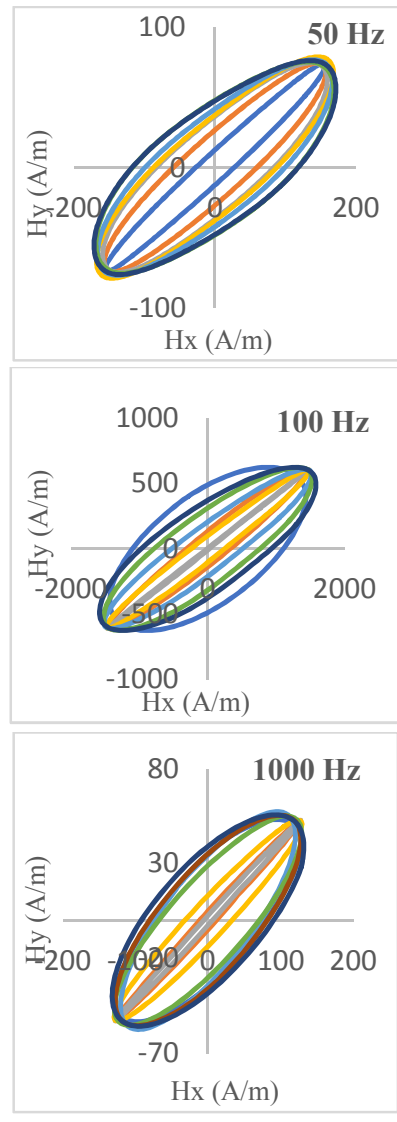

(a)

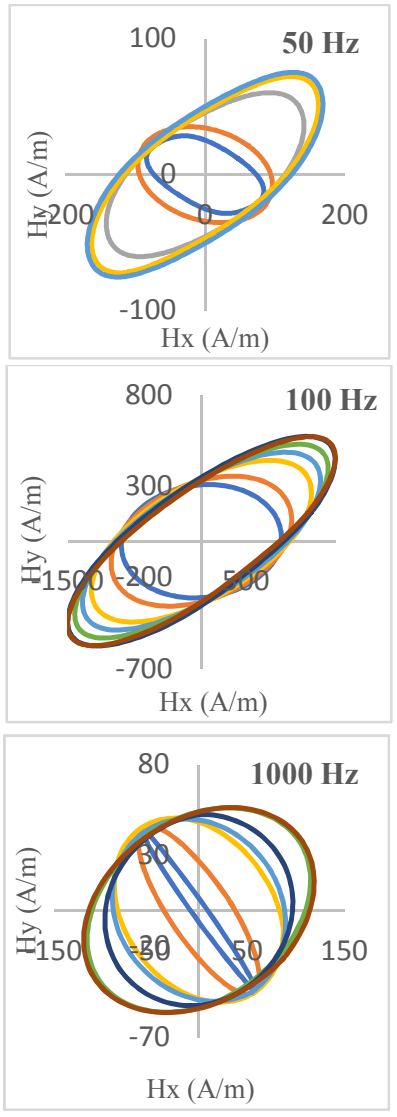

(b)
Fig. 6. $\mathrm{H}$ loci at $50 \mathrm{~Hz}, 100 \mathrm{~Hz}$ and $1000 \mathrm{~Hz}$ of operating frequency: a) $x$ major b) $y$-major

The performance of the SOMALOY 700 can be determined by referring to the core loss curve that has been shown in Fig. 7. It describes the total core loss for elliptical B loci (x or y major) at $50 \mathrm{~Hz}, 100 \mathrm{~Hz}$ and $1000 \mathrm{~Hz}$.

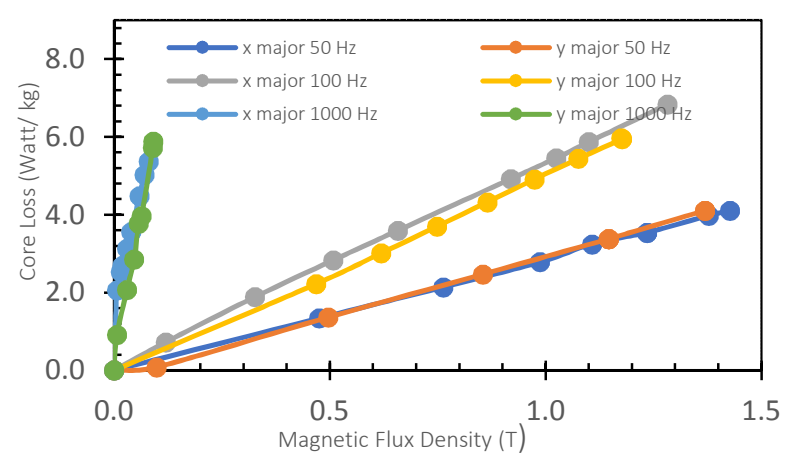

Fig. 7. Core loss of SOMALOY 700 material at 50, 100 and $1000 \mathrm{~Hz}$

\section{CONCLUSIONS}

The loci of magnetic flux density vector are controlled to be elliptical in order to resemble the actual magnetic flux excitation in rotating electrical machines. When there is some range of frequency, the core loss is higher at $1000 \mathrm{~Hz}$ compared to $50 \mathrm{~Hz}$ and $100 \mathrm{~Hz}$ due to the rotating domain particle during the magnetization process. In the future, the magnetic properties of this material will be measured up to 3$\mathrm{D}$ of magnetization phenomenon.

\section{REFERENCES}

[1] J.G. Zhu, J.J. Zhong, Z.W. Lin, and J.D. Sievert, "Measurement of core losses with 3D magnetic fluxes," IEEE Transactions on Magnetics, vol. 39, pp. 3429-3431, 2003.

[2] Z.W. Lin, H.W. Lu, J.G. Zhu, J.J. Zhong, X.L. Wang, and S.Y. Ding, "Vector characterization of soft magnetic materials," Journal of Applied Physics, vol. 97, pp. 10R306-1-3. 2005.

[3] Y.G. Guo, J.G. Zhu, Z.W. Lin, and J.J. Zhong, "3D vector magnetic properties of soft magnetic composite material," $J$. Magnetism and Magnetic Materials, vol. 302, no. 2, pp. 511-516, July 2006

[4] Y.G. Guo, J.G. Zhu, Z.W. Lin, J.J. Zhong, H.W. Lu, and S.H. Wang, "Calibration of sensing coils of a three-dimensional magnetic property tester," IEEE Transactions on Magnetics, vol. 42 , no. 10 , pp. $3243-3245$, Oct. 2006.

[5] Z.W. Lin, J.G. Zhu, Y.G. Guo, J.J. Zhong, and S.H. Wang, "Magnetic properties of soft magnetic composites under three dimensional excitations," Int. J. Applied Electromagnetics and Mechanics, vol. 25, pp. 237-241, 2007.

[6] Y.G. Guo, J.G. Zhu, Z.W. Lin, J.J. Zhong, H.Y. Lu, and S.H. Wang, "Determination of 3-D magnetic reluctivity tensor of soft magnetic composite materials," J. Magnetism and Magnetic Materials, vol. 312, pp. 458-463, May 2007.

[7] J. J. Zhong and J. G. Zhu, "Electromagnetic design of a 3D tester for magnetic properties of soft magnetic materials," in Proc. 5th Int. Conf. Elec. Machines and Syst., 2001, pp. 392 -395.

[8] Australian Motor, Operation Manual AM3002,

Available:

http://www.australianmonitor.com.au/assets/Uploads/Products/A ustralian-Monitor/Discontinued-Products/Australian-MonitorPre-2005---Manuals/AM3002-Manual.pdf [Accessed: 1-April2014]

[9] Y. Li, Q. Yang, J. Zhu, and Y. Guo, "Magnetic properties measurement of soft magnetic composite materials over wide range of excitation frequency," IEEE Trans. Ind. Appl., 2012.

[10] V. Basso and G. Bertotti, "Hysteresis in soft magnetic materials," J. Magn. Magn. Mat., vol. 215, pp. 1-5, 2000.

[11] Y. J. Li, Q. X. Yang, Y. H. Wang, J. G. Zhu, and Z. W. Lin, "Rotational core loss features of soft magnetic composite materials under excitation frequencies from $5 \mathrm{~Hz}$ to $1000 \mathrm{~Hz}$," in Proc. 2013 IEEE International Conference on Applied Superconductivity and Electromagnetic Devices, 2013, pp. . 